\title{
Electric field effects on the quantum efficiency of CsI photocathodes in gas media
}

\author{
A.Breskin, A.Buzulutskov* and R.Chechik \\ Department of Particle Physics \\ The Weizmann Institute of Science, 76100 Rehovot, Israel \\ D.Vartsky \\ Soreq Nuclear Research Centre, 70600 Yavne, Israel \\ G.Malamud ${ }^{\dagger}$ and P.Miné \\ LPNHE, Ecole Polytechnique, IN2P3-CNRS, 91128 Palaiseau, France
}

\begin{abstract}
We have measured the quantum efficiency (QE) of CsI photocathodes as a function of the electric field strength in a parallel-plate geometry, in $\mathrm{CH}_{4}, \mathrm{C}_{2} \mathrm{H}_{6}$ and $\mathrm{i}-\mathrm{C}_{4} \mathrm{H}_{10}$ both in charge collection and multiplication modes. It was found that in the collection mode the $\mathrm{QE}$ value in gases is lower compared to that of vacuum and is independent on the field; in gas media the QE starts to increase at the transition between collection and multiplication modes and reaches the vacuum value at high gas gain. We explain this effect by a decrease of the electron-molecule elastic backscattering while entering the multiplication mode. We conclude that the electric field effects observed here, would also apply for other photocathodes and gas mixtures. An enhancement of the QE after microdischarges was observed and is discussed in detail.
\end{abstract}

Submitted to Nuclear Instruments and Methods in Physics Research A

-on leave of absence from IHEP, Protvino, Russia

'presently at CERN, Geneva, Switzerland

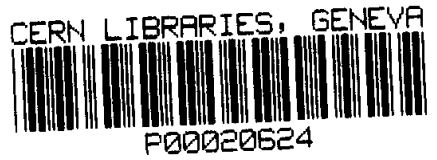




\section{Introduction}

The growing interest in UV-imaging detectors combining solid photocathodes with gaseous imaging electron multipliers has triggered a considerable experimental activity in photosensitive materials. CsI has been known since long as an efficient and relatively air-stable photoemissive material, used in vacuum-operated multipliers $[1,2]$. Following successfull attempts of operation under gas multiplication $[3,4]$ it has been systematically studied in recent years by several groups for its possible implementation in "gaseous photomultipliers" $[5,6,7,8]$. Its relatively high stability in air and under photon and ion bombardment has been proved [9] as well as its radiation hardness [10]. Methods of quantum efficiency (QE) enhancement after evaporation, by TMAE adsorption [5] and by heating the substrate [11] were proposed and further investigated [12]. However, serious discrepancies, of up to a factor 3, have appeared in the absolute values of the $\mathrm{QE}$ measured by different authors, in the wavelength range of $150-220 \mathrm{~nm}$. These discrepancies may have resulted from the different light flux calibration methods, methods of evaporation, methods of characterization, origin and crystalline form of the raw material [13], photon flux $[10,14,15]$ and polarization [14], post-evaporation treatment of the photocathode and other experimental conditions [16]. In adition, some authors measured higher $\mathrm{QE}$ in methane than in vacuum $[11,12]$.

One hypothesis for the discrepancies in the $\mathrm{QE}$ could be the value of the electric field, applied at the photocathode surface $[15,17]$. While some of the authors present QE data measured in a low electric field, in a charge collection mode, others measure the quantum response by counting photon-induced pulses resulting from an electron avalanche under a high electric field. In the present work the field effect on the QE has been investigated, for the first time, in a direct way.

We have studied the electric field dependence of the QE value of CsI in several hydrocarbon gases. We found that the QE strongly depends on the electric field in $\mathrm{C}_{2} \mathrm{H}_{6}$ and $\mathrm{i}-\mathrm{C}_{4} \mathrm{H}_{10}$ while in $\mathrm{CH}_{4}$ this dependence is very small. We attribute this dependence to the reduction of the photoelectron elastic backscattering probability with the commencement of inelastic collisions in the multiplication process. Sources of instability during data collection in CsI-based systems are discussed in detail. Since the field dependence of the QE value is mainly a function of the gas and the reduced electric field, we may conclude that this dependence could apply to any solid photoemissive material.

\section{Experimental set-up and procedures}

\subsection{Apparatus}

Three different sets of measurements were made in two different experimental arrangements. In the first set-up we have measured the relative $\mathrm{QE}$ in gases as a function of the 
electric field strength in charge collection and multiplication modes, using a single photon counting technique. In the same apparatus we have measured the gas gain curves, using a DC current recording technique. In the second set-up we have measured the absolute $\mathrm{QE}$ in vacuum and in gases in a collection mode, using a DC current technique.

A schematic view of the first set-up is shown in Fig.1. UV photons from a discharge $\mathrm{H}_{2}$ lamp, placed in air, enter the test chamber through a $\mathrm{CaF}_{2}$ window. The chamber can be either evacuated down to $10^{-5}$ Torr or flushed with gas under a controlled pressure. The light flux was reduced down to a single photon level by means of a collimator and two, $24 \mu \mathrm{m}$ thick, polypropylene film absorbers. Taking into account the $\mathrm{H}_{2}$ lamp emission spectrum, the film transmission and air cutoff, the average effective photon wavelength can be estimated to be of the order of $190 \mathrm{~nm}$.

A two-step parallel-plate structure, with a gap width of $3.2 \mathrm{~mm}$ each, was used for the photon detection: the photocathode, vacuum evaporated on a metallic plate, was followed by two wire mesh electrodes, $81 \%$ transparent. The photocathode and the second mesh were under negative and positive voltages respectively and the middle mesh was grounded. UV-induced photoelectrons from the photocathode were either collected in the first gap and multiplied in the second gap, or multiplied successively in both stages. The detector signal was measured in coincidence with a trigger pulse generated by the $\mathrm{H}_{2}$ lamp. In this set-up we studied the $\mathrm{CsI}$ photocathode response in three gases: $\mathrm{CH}_{4}$ and $\mathrm{C}_{2} \mathrm{H}_{6}$ at 60 Torr and $\mathrm{i}-\mathrm{C}_{4} \mathrm{H}_{10}$ at 20,40 , and 60 Torr.

The second experimental system consists of a high vacuum $\left(5 \cdot 10^{-8}\right.$ Torr $)$ evaporation chamber connected to a gas filling system and coupled via a $\mathrm{CaF}_{2}$ window to a vacuum monochromator equipped with a $\mathrm{D}_{2}$ lamp. This experimental system will be described in more detail elsewhere [18]. One of its important features is that the photocathode is prepared and brought into the measurement area in the same vacuum chamber, without contact to air. Our QE mearsurements were made using the DC current technique with the photocathode grounded through a picoampermeter and a positive voltage applied to a wire mesh, placed at a distance of $1.7 \mathrm{~mm}$.

All our CsI photocathodes were prepared in this system. The photocathodes mounted in the first set-up of Fig. 1 were exposed to air for a few minutes during the installation in the chamber. The photocathode preparation procedure includes the following stages: 1) $100 \mathrm{~nm}$ thick $\mathrm{Al}$ protective layer evaporation on a polished, preheated to $60^{\circ} \mathrm{C}$, stainless steel plate of $4 \mathrm{~cm}$ diameter; 2) $500 \mathrm{~nm}$ thick CsI layer evaporation; 3 ) "conditioning" of the photocathode at $60^{\circ} \mathrm{C}$ under high vacuum of $5 \cdot 10^{-8}$ Torr for a few hours $(2-10 \mathrm{~h})$. The last step was found necassary for a fast stabilization of the QE following the evaporation. In the second experimental system the $\mathrm{QE}$ measurements were carried out either at this temperature or at room temperature, without a noticeable difference, while in the first one they were all made at room temperature. 


\subsection{Measurement procedure}

\subsubsection{QE measurements using a pulse counting technique, and gain measure- ments}

The main advantage of the method used here for single photon counting is that the two-stage multiplication element allows one to maintain the same total gas gain while changing the field applied at the photocathode surface in the first gap. Thus a decrease of the amplification factor in the first gap is compensated by an increase of the gain in the second gap, and vice versa.

In the first set of measurements we measured the chamber detection efficiency, defined as the ratio of the number of coincidences between the chamber signal and the trigger, to the number of triggers. The trigger was provided by the $\mathrm{H}_{2}$ lamp pulse, at a rate equal to $60 \mathrm{sec}^{-1}$. To provide a single photoelectron operation mode, the intensity of the $\mathrm{H}_{2}$ lamp was reduced until the chamber efficiency was less than $20 \%$. From this efficiency we calculated the mean number of photoelectrons per light pulse. The chamber signal was processed by a preamplifier, a shaping amplifier and a discriminator. The amplifier shaping time was set to $5 \mu$ s to make the pulse shape, or the detection efficiency, independent of the gap at which the multiplication process takes place. A coincidence gate width of $10 \mu \mathrm{s}$ and a following dead time of $100 \mu \mathrm{s}$ were used in order to remove all short-term accidental coincidences, caused by photon and positive ion feedback from the avalanche. An accidental coincidences contribution to the chamber efficiency was negligible and was checked after each QE measurement by the replacement of the light collimator with an opaque screen.

In order to ensure the detection of photoelectrons with probability close to $100 \%$, the following procedure was used for each voltage on the first gap: the chamber detection efficiency was measured as a function of the voltage on the second gap, while maintaining the voltage on the first gap. The point at the middle of the efficiency plateau was chosen for the QE measurement. Working on the plateau garantees a full detection efficiency of photoelectrons escaping from the photocathode.

This statement was also confirmed by observing single photoelectron pulse height spectra obtained at the efficiency plateau as shown in Fig.2. They have a well defined peaked shape, which indicates the begining of the avalanche saturation. The spectra presented in Fig. 2 were measured at two extreme cases, namely for a low and a high field in the first gap, corresponding to the collection and multiplication modes. The spectra are almost identical, indicating full photoelectron collection in both cases.

For the measurements of the gain in the first gap we recorded the UV-induced DC current, substituting the pulsed $\mathrm{H}_{2}$ lamp by a continuous flux low-pressure $\mathrm{Ar}(\mathrm{Hg})$ lamp. The picoampermeter was connected to the middle mesh. 


\subsubsection{QE measurements using a current technique}

Absolute QE measurements were carried out in vacuum and in gases, using our photocathode preparation/characterization system, in the wavelength range of $150-220 \mathrm{~nm}$. After preparation of the CsI photocathode according to the procedure described in section 2.1, the electrode plate with the CsI layer was displaced within the vacuum chamber, from the evaporation area to the measurement area. The photocathode was exposed to a photon flux of typically $10^{6}$ photons $/ \mathrm{sec} \cdot \mathrm{mm}^{2}$. The photocurrent was measured on the photocathode, and a positive voltage was applied on the mesh electrode. The electric field strength values in the gases were chosen such as to be in the collection mode, i.e. in the middle of the QE plateau, measured with the pulse counting technique and discussed in section 3.2 and 3.3. In vacuum, the current plateau extends over a large range of the electric field and typically we applied $600 \mathrm{~V}$ on the $1.7 \mathrm{~mm}$ gap. An absolute light flux calibration of the monochromator beam was made with the help of a vacuum photodiode, calibrated by the National Institute of Standards and Technology (NIST) of the US Department of Commerce. It was used to recalibrate our reference photomultipliers, as discussed in section 3.3 .

The measurement procedure was as follows: the QE was measured in vacuum (better than $10^{-7}$ Torr); a gas was flushed through the chamber under controlled pressure and the QE was measured; the chamber was evacuated during several hours and the QE was measured in vacuum again. This procedure was repeated for all three gases investigated. The purity of the gases used was at the level of a few ppm. Before introduction into the vacuum chamber, the gas was flushed through a gas filling system during several hours, while monitoring its oxigen and water levels.

\section{Experimental results}

\subsection{Breakdowns and stability problem}

It turned out that one of the most delicate problems encountered in the pulse counting measurements was an instability caused by microdischarges or small breakdowns. Similar to ref.[19], where breakdowns in a parallel-plate structure have been studied in detail, we observed two well distinguished types of breakdowns: "fast" and "slow". The "fast" breakdown is self-quenched; on the oscilloscope it looks like a single pulse of high amplitude accompanied by a current jump. Following such events, the chamber looses its efficiency for several seconds. About 30 seconds are needed for full efficiency recovery. "Fast" breakdowns took place occasionally while working in a collection mode in the first gap. The most probable reason for them is a spark breakdown in the second gap (operating at high gain).

The "slow" breakdowns arised when operating the first gap in a multiplication mode. 
They appear like a continuous discharge resulting in a high pulse rate, lasting for several seconds. In $\mathrm{C}_{2} \mathrm{H}_{6}$ and $\mathrm{i}-\mathrm{C}_{4} \mathrm{H}_{10}$ they were accompanied by high current jumps and usually, but not always, resulted in automatically switching off of the voltage power supply. In $\mathrm{CH}_{4}$ the current jumps were not so well defined, and the only signature of a "slow" breakdown was the oscilloscope signal. We do not know whether it is related to the $\mathrm{CH}_{4}$ itself, or to the degradation of the photocathode, since the measurements of detection efficiency with $\mathrm{CH}_{4}$ were made after the measurements of the gain, i.e. after exposing the photocathode to an intense light flux. It is interesting to note that the "slow" breakdowns appeared just after the beginning of the multiplication in the first gap and had a maximum probability (about one breakdown per 5 minutes) at moderate gains. For the highest gain $\left(10^{5}-10^{6}\right)$ in the first gap the probability of "slow" breakdowns dropped considerably. Thus, the two marginal cases, collection and high multiplication in the first gap have the best stability.

It should be remarked that after a "slow" breakdown we have noticed a considerable enhancement of the $\mathrm{QE}$ value (a contribution from accidental coincidences was measured to be negligible), which ammounted to few tens of percents. This phenomenon was most pronounced at low (10 Torr) pressure. It is illustrated in Fig.3, showing the history of the number of photoelectrons per light pulse extracted from the CsI photocathode. The measurements were made with 10 Torr $\mathrm{i}-\mathrm{C}_{4} \mathrm{H}_{10}$ gas filling and with a reduced electric field strength of 100 and $250 \mathrm{~V} / \mathrm{cm}$. Torr in the first and second gap respectively. One can see that after a "slow" breakdown the QE increases dramatically, and then gradually decreases to the initial value with a time constant of about 3 minutes.

Replacing the gas volume by a fast evacuation followed by a new gas filling has been proved to be the only way to stabilize the chamber operation and to reproduce the data after a "slow" breakdown. For $\mathrm{CH}_{4}$ a $\mathrm{QE}$ increase with time was observed even without noticeable "slow" breakdowns; sometimes it happened after long-term operation in a multiplication mode in the first gap. Again, a fast gas replacement, including the evacuating stage, immediatelly restored the data stability.

Most probably the "slow" breakdown is caused and sustained by photon feedback from the excited radicals in the first gap interacting with the photocathode [19]. We interpret the post-breakdown QE enhancement as a result of the well known Malter effect [20]: electron extraction by very high local electric field created by positive ions deposited on the insulator surface. We will discuss this subject in more detail in section 4.2.

Based on our experience, we deduced the following rules to obtain reproducible data:

- After switching on the voltage in the photocathode gap one should wait for about 5 minutes before starting the measurements, in order to let the upcharging and polarization processes to be completed.

- While working in a collection mode in the photocathode gap, one should watch for "fast" breakdowns, and if such has happened, interrupt the measurment for about $30 \mathrm{sec}$. 
- While working in a multiplication mode in the photocathode gap, one should watch for "slow" breakdowns, and if it has happened, evacuate the gas from the chamber, refill it with a new gas and repeat the last measurement.

- While working in a multiplication mode in the photocathode gap, one should accept data only after checking their stability during at least $5 \mathrm{~min}$. In case of instability the gas volume should be replaced using the evacuating/refilling procedure.

\subsection{QE of CsI in gases obtained with the pulse counting tech- nique}

Fig.4 shows the number of photoelectrons per light pulse (which is proportional to the $\mathrm{QE}$ ) as a function of the electric field strength in the photocathode gap, measured with i$\mathrm{C}_{4} \mathrm{H}_{10}$ at three different pressures. The features common to all pressures are the following: at very low fields the QE increases with the electric field and then it reaches a plateau. The plateau ends at some transition point and the QE further increases. At 20 and 40 Torr one may even notice a possible saturation of the $Q E$ at the last points. The points at which the plateau ends depend on the gas pressure: the higher the pressure, the larger the electric field at this transition point. But as it will be made clear from the following, this point is invariant for all pressures in the $\mathrm{E} / \mathrm{p}$ (reduced electric field) scale.

Figs. 5 and 6 show the relative QE for the three gases investigated, as a function of the reduced electric field. The data points are normalized to the middle of the platean, since for all gases and pressures investigated the QE plateau obviousely exists. The error bars presented in the figures are the statistical errors. The systematic errors are mainly due to the stability problem, discussed in the previous section. On the plateau we estimate the systematic errors to be smaller than the statistical errors, while in the other region their values vary from gas to gas and can be as large as $10 \%$ for $\mathrm{i}-\mathrm{C}_{4} \mathrm{H}_{10}$ and $2-3 \%$ for $\mathrm{CH}_{4}$.

The curves of the gas gain in the photocathode gap are plotted on the same figures. In fact, what is called "gain", is a current, recordered in the first gap and normalized to the value at the plateau. In contrary to a typically flat QE plateau measured in the pulse counting mode, a current plateau is not exactly flat, as observed by several authors. Therefore we normalized the "gain" to the midpoint of the plateau. We suppose that the reason for the non-flatness of the plateau is that in the current technique a total current is recorded, including the part caused by photon feedback due to excitation processes during the photoelectron drift (in the pulse counting technique these processes are discarded by the dead-time gate). The QE dependence on the field also gives a small contribution to the measured current. Thus the real gas gain values may be overstimated, but probably by less than by a factor of two.

Looking at the correlation between the QE and the gain dependence on the electric field in $\mathrm{i}-\mathrm{C}_{4} \mathrm{H}_{10}$, one can make two important observations: 1) the QE plateau corresponds 
to a charge collection mode in the photocathode gap; 2) for all pressures the begining of the QE increase takes place approximately at the same value of the reduced electric field, of about $40-50 \mathrm{~V} / \mathrm{cm}$. Torr, which corresponds to the transition from the collection to the multiplication mode.

Fig.6 shows the relative $\mathrm{QE}$ and the gain curves, measured at 60 Torr $\mathrm{C}_{2} \mathrm{H}_{6}$ and $\mathrm{CH}_{4}$. In general the $\mathrm{QE}$ behaviour with field for these two gases is the same as for $\mathrm{i}-\mathrm{C}_{4} \mathrm{H}_{10}$. The $\mathrm{QE}$ has a well defined plateau in the collection mode and starts to increase at about 40 $\mathrm{V} / \mathrm{cm}$. Torr, which corresponds to the begining of the multiplication mode. For $\mathrm{i}-\mathrm{C}_{4} \mathrm{H}_{10}$ and $\mathrm{C}_{2} \mathrm{H}_{6}$ a rather fast increase in $\mathrm{QE}$ of $10-15 \%$ occurs at the transition point, which is not the case in $\mathrm{CH}_{4}$.

We conclude that, qualitatively, the $\mathrm{QE}$ dependence on the field exists in all gases. The only difference is in the amplitude of the effect, i.e. in the ratio of the QE value at the highest multiplication to that of the collection mode. This ratio is largest for $\mathrm{i}-\mathrm{C}_{4} \mathrm{H}_{10}(30$ $40 \%)$, lower for $\mathrm{C}_{2} \mathrm{H}_{6}(20-25 \%)$, and considerably lower for $\mathrm{CH}_{4}(3-5 \%)$. The "universal" behaviour of the QE for the different gases and pressures is illustrated in Fig.7, where the relative QE data points from Figs.5,6 are displayed in the same plot. The two regimes of the QE dependence on the electric field, namely the collection plateau and the growth with the gain, are clearly distinguishable. A transition point between these two regimes is well pronounced.

\subsection{QE of CsI in vacuum and gases obtained with current tech- nique}

We have measured the absolute $\mathrm{QE}$ in collection mode in vacuum and in $\mathrm{CH}_{4}, \mathrm{C}_{2} \mathrm{H}_{6}$ and $\mathrm{i}-\mathrm{C}_{4} \mathrm{H}_{10}$ at 60 Torr. The electric field strength in vacuum was $2.9 \mathrm{kV} / \mathrm{cm}$ and in gases $1.8 \mathrm{kV} / \mathrm{cm}$, which corresponds to the middle of the plateau in the $\mathrm{QE}$, measured by the pulse counting technique.

Fig.8 shows the QE plots of some CsI photocathodes, recently measured in vacuum in our set-up [18]. These values are considerably higher than that published in our earlier works $[10,12,17,21]$. This discrepancy is due to the wrong values, supplied by the manufacturer, of the QE of photomultipliers used as a reference for the light flux in these works [22].

The ratio of the QE values in vacuum to that of gases is shown in Fig.9. In accordance with recently published results [10] we found, when carefully adjusting the electric field value to a collection mode, that the $\mathrm{QE}$ value in gases is always lower than in vacuum. For $\mathrm{CH}_{4}$ at $190 \mathrm{~nm}$ the difference is small, and is about $3 \%$, while for the other two gases it is considerably larger: close to $20 \%$ for $\mathrm{C}_{2} \mathrm{H}_{6}$ and to $30 \%$ for $\mathrm{i}-\mathrm{C}_{4} \mathrm{H}_{10}$. The behaviour of the curves for the wavelengths shorter than $175 \mathrm{~nm}$ is not related to a QE drop, but is caused by the different VUV light absorption in the hydrocarbons investigated. The relative decrease in the QE in gases at wavelengths above $190 \mathrm{~nm}$ could have resulted either from 
a higher dependence of the $\mathrm{QE}$ on the electric field at low photoelectron energies or from a larger systematic error due to the lower light intensity. This will be further investigated.

When comparing the two sets of the QE data, obtained in two different experimental set-ups and with two different techniques, one can notice a strong coherence between them. The ratio of the maximum $\mathrm{QE}$ value in the multiplication mode to that of the collection mode, measured in the first set-up for a given gas, is close to the ratio of the $\mathrm{QE}$ in vacuum to the $\mathrm{QE}$ in gas, measured in the collection mode in the second set-up. This leads us to the hypothesis, that the maximum $Q E$ value in gases, while working in a multiplication mode, corresponds to the $\mathrm{QE}$ value in vacuum.

\section{Discussion}

\subsection{The electric field effect on the QE of CsI in gases}

The interpretation presented below is based on the assumption that the QE in gases is always lower compared to vacuum, due to the photoelectron backscattering caused by elastic collisions between the photoelectrons and the gas molecules. This well known $\mathrm{QE}$ reduction effect is large especially for noble gases $[23,24,5]$. Due to the large mass difference between an electron and a molecule, elastic collisions between them will cause the photoelectron back diffusion to the photocathode [25]. This is the situation in the collection mode.

Under high electric field the energy of the photoelectron becomes high enough to excite and to ionize the molecules of the gas, and the probability for elastic scattering decreases due to the opening of new, inelastic, channels in the electron-molecule collisions. This happens as soon as multiplication mode starts. The inelastic channels do not lead to backscattering, since the photoelectron looses its energy without a substantial change of its direction of motion. When the probability of the elastic collisions drops down to zero, the $\mathrm{QE}$ in gases must reach the vacuum value.

Fig.10 (from ref.[25]) provides an example of the fraction of energy losses for different processes as a function of the reduced electric field for $\mathrm{Ar}, \mathrm{N}_{2}$ and $\mathrm{H}_{2}$. With the increase of the field the elastic process is taken over by the molecular excitation and the ionization processes, as discussed above. Note, that a comparatively smaller energy fraction for elastic collisions in molecular gases does not necassary mean their smaller probability to occur, since the energy transfer in each elastic collision is small. The field value at which the elastic process probability drops down and the ionization takes over is about $40 \mathrm{~V} / \mathrm{cm}$. Torr. This is very close to the value we observed in hydrocarbons.

This picture can also explain the fast rise in $Q E$ in $\mathrm{C}_{2} \mathrm{H}_{6}$ and $\mathrm{i}-\mathrm{C}_{4} \mathrm{H}_{10}$ mentioned in section 3.2: it could have been induced by a resonance dependence of the molecular excitation cross-section on the energy (see for example figure for $\mathrm{CO}_{2}$ in ref.[26]). Assuming that our present interpretation is correct, we state the following: 
- The field effect on the QE is mainly influenced by the nature of the gas and should be practically independent of the nature of the photocathode. The photocathode properties could have a small influence depending on the photoelectron energy.

- A similar field effect should also exist for noble gases, since the basic collision processes are common for all gases. This in fact is evident from Fig.10.

- The larger is the fraction of the elastic scattering in the low field region (collection mode), the larger should be the QE increase when going to the high field region (multiplication mode). Since for noble gases the fraction of the elastic scattering is large (up to 100\%) in the low field region, the field-induced QE enhancement must be most prominent.

Since the first works on gaseous photodiodes, it was believed that solid photocathodes cannot be used with noble gases due to a considerable drop of the QE as compared to vacuum [24]. However our prediction leads to the conclusion, that while working in a multiplication mode, one can obtain, in noble gases or their mixtures with appropriate quencher, a $\mathrm{QE}$ close to the vacuum value, provided a high reduced electric field can be reached. Consequently this may revive the interest in noble gas-filled photodiodes with solid photocathodes in the visible range. An advantage of noble gases is that in contrary to organic gases, they do not interact with or destroy visible-range photocathodes. High electric field conditions for pure noble gases can be obtained, for example, by operating in a pulsed voltage supply mode.

Recent observation in ref. [27] tends to agree with our predictions. These authors observed, in collection mode, a $50 \%$ drop of the $\mathrm{QE}$ in 50 Torr $\mathrm{i}-\mathrm{C}_{4} \mathrm{H}_{10}$ as compared to vacuum. This is close to our result despite of the different photocathode type and the wavelength.

\subsection{The QE enhancement of CsI after microdischarges}

The phenomenon of QE enhancement after the treatment of the photocathode by a discharge is known since early works on gas-discharge counters. As for example, a corona discharge treatment of metallic photocathodes in Geiger counters was found in early works to increase the $\mathrm{QE}$ by a large factor and made it sensitive to visible light. Recently the QE enhancement of the CsI-TMAE photocathode under discharge conditions has been reported [28]. In spite of the different conditions, the common feature in all these works including the present one is that the detector evacuation eliminated the QE enhancement, caused by a discharge. It has been understood that positive ions play a substential role in the enhancement effect after breakdowns [28] and under high photon flux conditions [14], but its mechanism is still unclear.

We suppose that this mechanism is related to the Malter effect [20]: an electron emission from a dielectric layer, caused by a strong electric field (hundreds of kilovolts 
per $\mathrm{cm}$ ). In gas-discharge counters it arises when the cathode has regions covered with an insulator film [29]: positive ions from an avalanche are deposited on the insulator surface and thus cannot be neutralized. They create an electric field which is strong enough to considerably increase the electron emission probability.

We suppose that this effect, in our case, increases the photoelectron emission probability and shifts the red boundary of the photoeffect, in a similar way to the Schottky effect in metals (metal work function decrease due to a very high electric field). The Malter effect could be applied here despite the fact that CsI in thin films is slightly conductive. Indeed the bulk CsI is a natural insulator, but in thin layers it conducts electricity. Its conductivity could be due to metal islands of $\mathrm{Cs}$ on the surface (recently an excess of Cs on CsI surface was reported [30]), or other surface effects like adsorbed monolayers of water. At present we can only speculate on this matter. However, since CsI is an insulator, there should exist nonconductive regions on its surface, where the Malter effect could indeed take place.

Following these arguments it is easier to understand a discharge-induced QE enhancement of CsI: after "slow" breakdowns or under high flux conditions, a concentration of positive ions on the nonconductive surface regions of CsI may become large enough to create a high electric field within the CsI film. This could be responsible for the increase of the photoelectron extraction probability. Positive ions deposited on such regions can be gradually neutralized by charge transfer processes to the nearby conductive regions, for example by a charge exchange reaction with gas molecules. The most effective way to remove them is to evacuate the chamber.

Charge transfer processes between the deposited positive ions and the molecules of the gas are more effective at higher pressures. The neutralization process can also depend on the nature of the gas. It could explain for example why the post-breakdown QE enhancement is more pronounced at 10 Torr $\mathrm{i}-\mathrm{C}_{4} \mathrm{H}_{10}$ and less important at higher pressures.

\section{Conclusions}

The results of the present work may be summarized as follows:

- We have measured relative $\mathrm{QE}$ value of CsI photocathodes as a function of the electric field strength in a parallel-plate geometry, in $\mathrm{CH}_{4}, \mathrm{C}_{2} \mathrm{H}_{6}$ and $\mathrm{i}-\mathrm{C}_{4} \mathrm{H}_{10}$ both in collection and multiplication modes using a pulse counting technique. We have measured in addition the QE of CsI in these gases and in vacuum in a collection mode, using a current technique.

- Combining our results from pulse counting and from current recording in the collection mode we conclude that the QE value in gases is lower compared to that of 
vacuum and is independent of the field when working in the collection plateau. For $\mathrm{CH}_{4}$ this difference is small, of the order of $3 \%$, while for $\mathrm{C}_{2} \mathrm{H}_{6}$ and $\mathrm{i}-\mathrm{C}_{4} \mathrm{H}_{10}$ it is large, reaching $20-30 \%$.

- In gas media the $\mathrm{QE}$ value starts to increase at the transition between the collection and multiplication modes and reaches the vacuum value at high gas gain. We explain this effect by a decrease of the electron-molecule elastic backscattering probability as multiplication starts.

- It was observed that the QE value of CsI can considerably increase after microdischarges, which results in data instability. We explain this effect by a positive ion surface deposition on the nonconductive regions of CsI, which create a very high local electric field and thus increase the photoelectron extraction probability. We propose some safety measures to be taken during measurements, before new data collection.

Our results suggest that:

- Unless $\mathrm{CH}_{4}$ is used as the detector gas, a UV-detector based on a parallel-plate electron multiplier may have an advantage over that having a multiwire multiplier. This is due to a considerable increase of the $Q E$, resulting from the higher electric field at the photocathode, reached in the parallel-plate geometry for an equal gain. On the other hand one should take into account, that for the same reason the energy of positive ions hitting the photocathode is higher in the parallel-plate structure, which could result in its faster degradation. A two-step detector could solve this problem. One should also take into account that a parallel plate structure is less stable against discharges, and that these discharges may strongly modify the photocathode response.

- One can use in principle any gas filling, including mixtures based on noble gases, and in combination with any solid photocathode, provided the electric field at the photocathode is sufficiently high to allow for an efficient photoelectron extraction.

According to our understanding the QE data of CsI of various groups, obtained by current measurements in vacuum and in $\mathrm{CH}_{4}$ and by the pulse counting method in various gases, should be similar to each other and larger than those obtained by current measurements in hydrocarbons other than $\mathrm{CH}_{4}$. However, this is not the case. The discrepancies between QE data of different groups are only partially explained by the electric field effects discussed here, and by possible instabilities and microdischarges during the measurements. Obviously, other important factors may influence the QE of CsI. Among them are surface effects related to the evaporation procedure (the crystalline form of the 
raw material, impurities, temperature) and to the incident photon flux and polarization. Some of these phenomena are presently investigated by different groups.

\section{Acknowledgements}

We would like to thank Drs. G.Paic, F.Piuz and J.Va'vra for stimulating discussions.

This work was supported by the Foundation Mordoh Mijan de Solonique, by the Basic Research Foundation of the Israel Academy of Science and Humanity, and by the United State-Israel Binational Science Foundation (BSF). A.Buzulutskov is grateful to the Feinberg Graduate School for his support.

\section{References}

[1] G.R.Carruthers, Appl. Opt., 14 (1975) 1667.

[2] D.G.Simons, G.W.Fraser, P.A.J.DeKorte, J.F.Pearson and L.DeJong, Nucl. Instr. and Meth. A261 (1987) 579.

[3] V.Dangendorf, A.Breskin, R.Chechik and H.Schmidt-Böcking, Nucl. Instr. and Meth. A289 (1990) 322.

[4] G.Charpak, V.Peskov, D.Scigocki and V.Valbis, Proc. Int. Simp. on Particle Identification at High Luminosity Hadron Colliders (ed. T.G.Gouraly and I.G.Morfin), FNAL, IL, USA (1989) 295.

[5] J.Seguinot, G.Charpak, Y.Giomataris, V.Peskov, J.Tischhauser and T.Ypsilantis, Nucl. Instr. and Meth. A297 (1990) 133.

[6] B.Hoeneisen, D.F.Anderson and S.Kwan, Nucl. Instr. and Meth. A302 (1991) 447.

[7] M.Staric, A.Stanovnik and P.Krizan, Nucl. Instr. and Meth. A300 (1991) 213.

[8] See list of references to other works in P.Miné, Photoemissive materials and their application to gaseous detectors, Preprint X-LPNHE/93-09, Proc. of the First Workshop on RICH Detectors, Bari, Italy, June 2-5, 1993. To be published in Nucl. Instr. and Meth. A.

[9] V.Dangendorf, A.Breskin, R.Chechik and H.Schmidt-Böcking, Nucl. Instr. and Meth. A308 (1991) 519.

[10] G.Malamud, P.Miné, D.Vartsky, A.Breskin and R.Chechik, Nucl. Instr. and Meth. A335 (1993) 136. 
[11] D.F.Anderson, S.Kwan, V.Peskov and B.Hoeneisen, Nucl. Instr. and Meth. A323 (1992) 626.

[12] H.Brauning, A.Breskin, R.Chechik, P.Miné and D.Vartsky, Nucl. Instr. and Meth. A327 (1993) 369.

[13] D.F.Anderson, S.Kwan and V.Peskov, Nucl. Instr. and Meth. A326 (1993) 611.

[14] A.Braem, A.DiMauro, E.Nappi, A.Ljubicic, G.Paic, F.Piuz, F.Poza, R.S.Ribeiro, T.Scognetti, T.D.Williams, Fast RICH detector with a cesium iodide photocathode at atmospheric pressure, Preprint CERN/PPE/93-209, Proc. of the First Workshop on RICH Detectors, Bari, Italy, June 2-5, 1993. To be published in Nucl. Instr. and Meth. A.

[15] D.F.Anderson, S.Kwan and V.Peskov, CsI and some new photocathodes, Preprint Fermilab-Conf-93/128, Proc. of the First Workshop on RICH Detectors, Bari, Italy, June 2-5, 1993. To be published in Nucl. Instr. and Meth. A.

[16] See related articles in the Proc. of the First Workshop on RICH Detectors, Bari, Italy, June 2-5, 1993. To be published in Nucl. Instr. and Meth. A.

[17] G.Malamud, P.Miné, D.Vartsky, B.Equer, A.Breskin and R.Chechik, The photoyield of Csl, amorphous silicon and organometallic reflective photocathode materials, Preprint X-LPNHE/93-10, Proc. of the First Workshop on RICH Detectors, Bari, Italy, June 2-5, 1993. To be published in Nucl. Instr. and Meth. A.

[18] A.Breskin, A.Buzulutskov, R.Chechik, H.Brauning, D.Vartsky, G.Malamud and P.Miné, CsI studies with a novel system designed for preparation and characterization of air-sensitive photoemissive materials. In preparation.

[19] P.Fonte,V.Peskov and F.Sauli, Nucl. Instr. and Meth. A305 (1991) 91.

[20] L.Malter, Phys. Rev. 49 (1936) 876.

[21] G.Malamud, P.Miné, D.Vartsky, B.Equer, P.Besson, Ph.Bourgeois, A.Breskin and R.Chechik, Measurements of the quantum efficiency of CsI, amorphous silicon and organometallic photocathodes, Preprint X-LPNHE/93-13, presented at the Third London Conf. on Position Sensitive Detectors, September 1993. To be published in Nucl. Instr. and Meth. A.

[22] A.Breskin, R.Chechik, D.Vartsky, G.Malamud and P.Miné, A correction of the quantum efficiency of CsI and other photocathodes due to the recalibration of the reference photomultipliers, Preprint WIS-93/116/Dec.-PH. Submitted to Nucl. Instr. and Meth. A. 
[23] See for example D.F.Anderson, R.Bouclier, G.Charpak, S.Majewski and G.Kneller, Nucl. Instr. and Meth. 217 (1983) 217.

[24] G.Charpak, W.Dominik, F.Sauli, S.Majewski, IEEE Trans. Nucl. Sci. NS-30 (1983) 134.

[25] L.B.Leob, Basic processes of gaseous electronics, University of California Press, Berkley, 1961.

[26] V.Palladino and B.Sadoulet, Nucl. Instr. and Meth. 128 (1975) 323.

[27] E.Gushchin, A.Lebedev, S.Somov et al., Pribory i Technika Experimenta, N5 (1993) 143 (in Russian).

[28] S.Kwan and D.F.Anderson, Nucl. Instr. and Meth. A309 (1991) 190.

[29] F.Sauli, Principle of operation of multiwire proportional and drift chambers, in: "Experimental Techniques in High Energy Physics" (ed. T.Ferbel), Addison-Wesley Publ. Co., 1987.

[30] C.Coluzza, J.Ameida, H.Berger, L.Perez, G.Margaritondo, G.Paic, A.Braem, F.Piuz, A.DiMauro and E.Nappi, Spatially resolved X-ray spectroscopy of CsI deposits, Proc. of the First Workshop on RICH Detectors, Bari, Italy, June 2-5, 1993. To be published in Nucl. Instr. and Meth. A. 


\section{Figure captions}

Fig.1 A schematic view of the first experimental set-up for the field-dependence study of the quantum efficiency. UV-induced photoelctrons are multiplied in a two-stage geometry. The relative quantum efficiency is measured in a pulse counting mode (see text).

Fig.2 Single photoelectron pulse height spectra, obtained in the set-up shown in Fig.1, with low (a) and high (b) electric field (indicated in the figure) in the photocathode gap, in 40 Torr $\mathrm{i}-\mathrm{C}_{4} \mathrm{H}_{10}$. The total gain is of the order of $10^{6}$.

Fig.3 The number of photoelectrons per light pulse emitted from a CsI photocathode in 10 Torr $\mathrm{i}-\mathrm{C}_{4} \mathrm{H}_{10}$ as a function of time. Arrows indicate the moment at which "slow" breakdowns occured.

Fig.4 The number of photoelectrons per light pulse emitted from a CsI photocathode at different pressures of $\mathrm{i}-\mathrm{C}_{4} \mathrm{H}_{10}$ as a function of the electric field strength.

Fig.5 The relative quantum efficiency of CsI and the gas gain, as a function of the reduced electric field in $\mathrm{i}-\mathrm{C}_{4} \mathrm{H}_{10}$ at pressures of 20 Torr (a), 40 Torr (b) and 60 Torr (c). The $\mathrm{QE}$ data points are normalized to the middle of the plateau in the collection mode.

Fig.6 The relative quantum efficiency of $\mathrm{CsI}$ and the gas gain, as a function of the reduced electric field in 60 Torr of $\mathrm{C}_{2} \mathrm{H}_{6}$ and $\mathrm{CH}_{4}$. The $\mathrm{QE}$ data points are normalized to the middle of the plateau in the collection mode.

Fig.7 The relative quantum efficiency of CsI as a function of the reduced electric field for all gases and pressures investigated.

Fig.8 The absolute quantum efficiency of CsI, in vacuum, as a function of the wavelength. Shown in the figure are results of one of our best photocathodes and a mean curve based on 5 different photocathodes.

Fig.9 The ratio of the vacuum quantum efficiency of a CsI photocathode to the quantum efficiency measured in $\mathrm{CH}_{4}, \mathrm{C}_{2} \mathrm{H}_{6}$ and $\mathrm{i}-\mathrm{C}_{4} \mathrm{H}_{10}$, as a function of the wavelength.

Fig.10 The fraction of the energy going into different processes in argon, nitrogen and hydrogen as a function of the reduced electric field. El represents the elastic collisions, EV the vibration excitations, EE the electron excitations, $\mathrm{E}$ the ionizations and $S$ the increase of the electron kinetic energy. Figure taken from ref.[25]. 


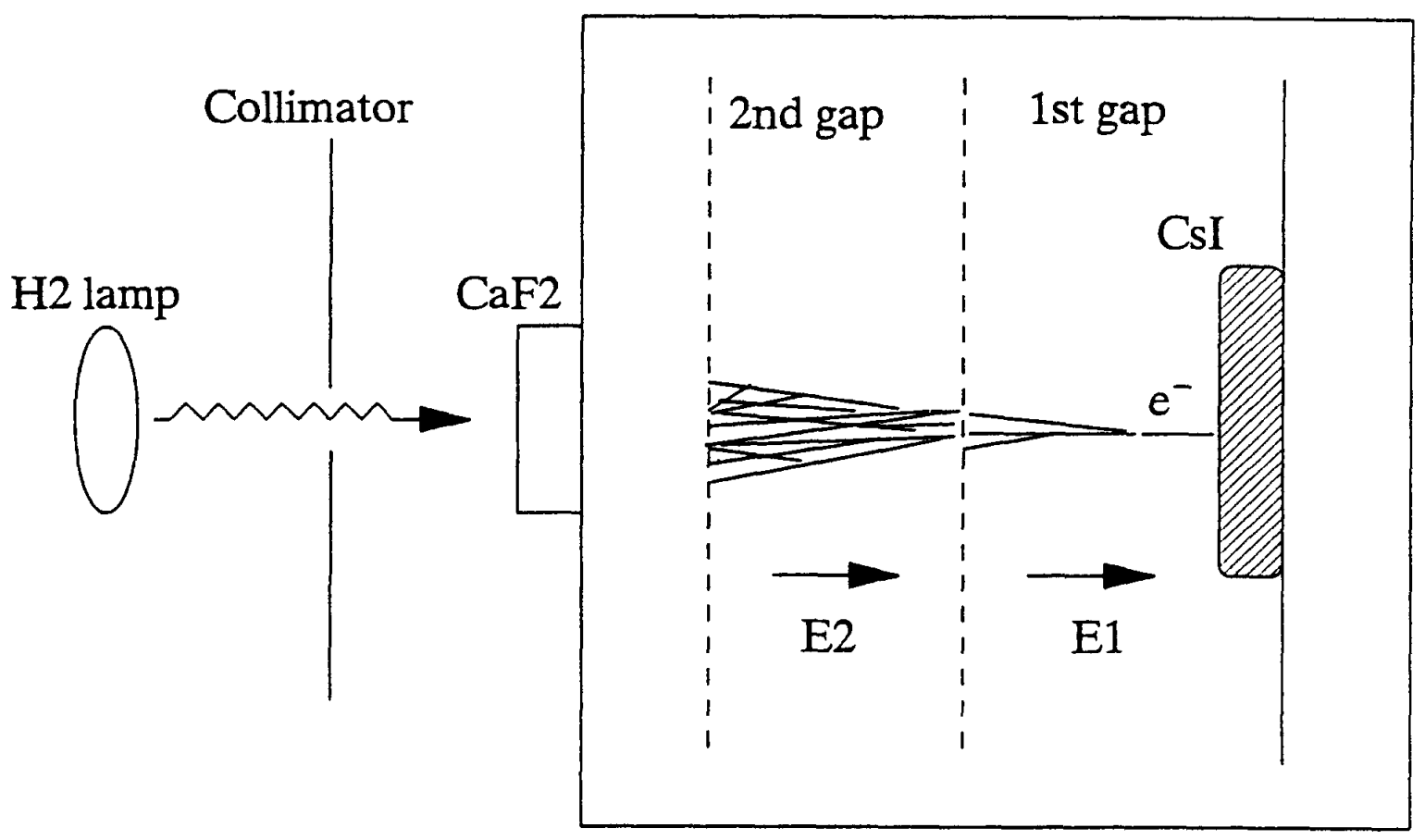

Fig. 1 

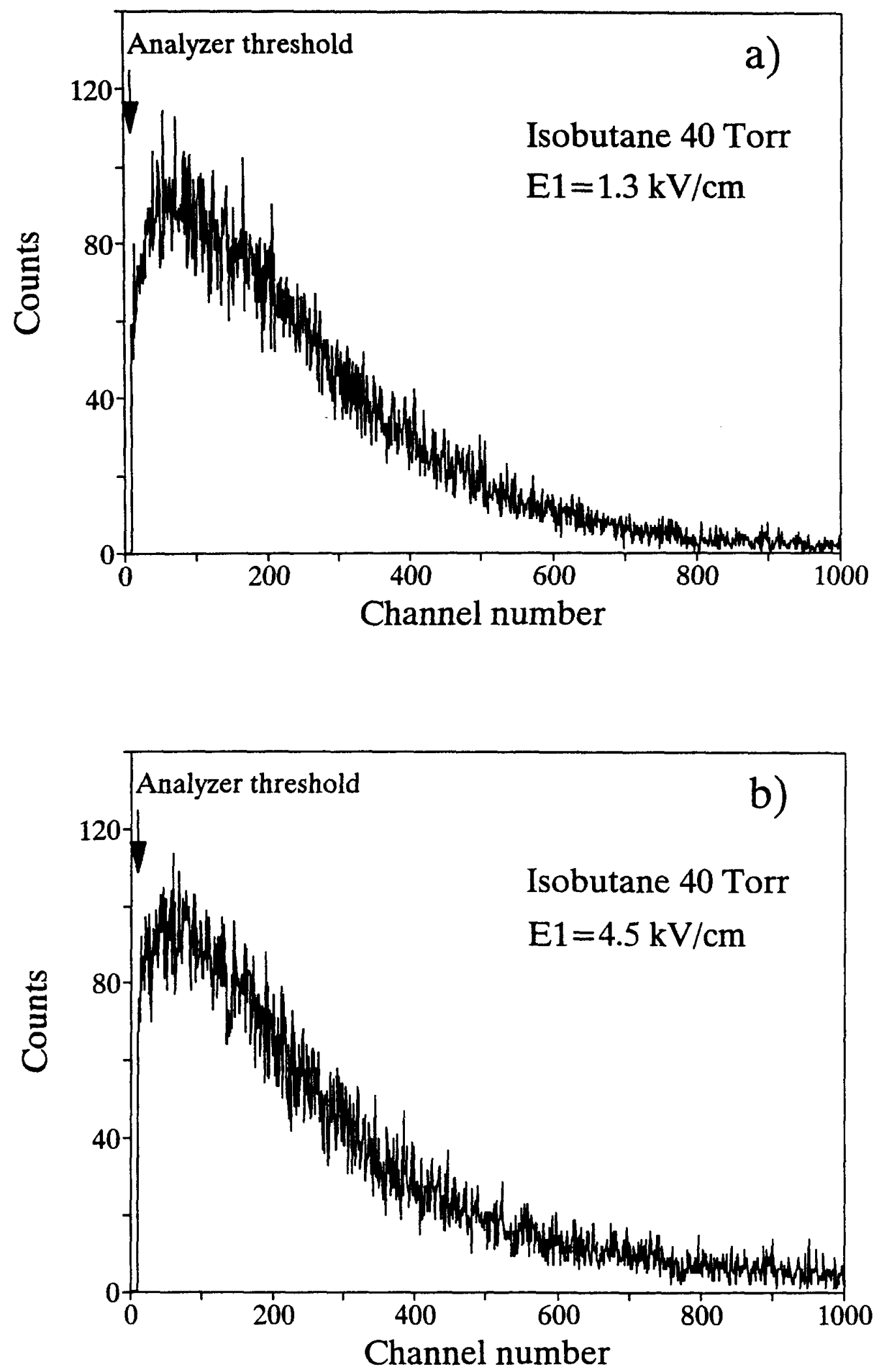

Fig. 2 


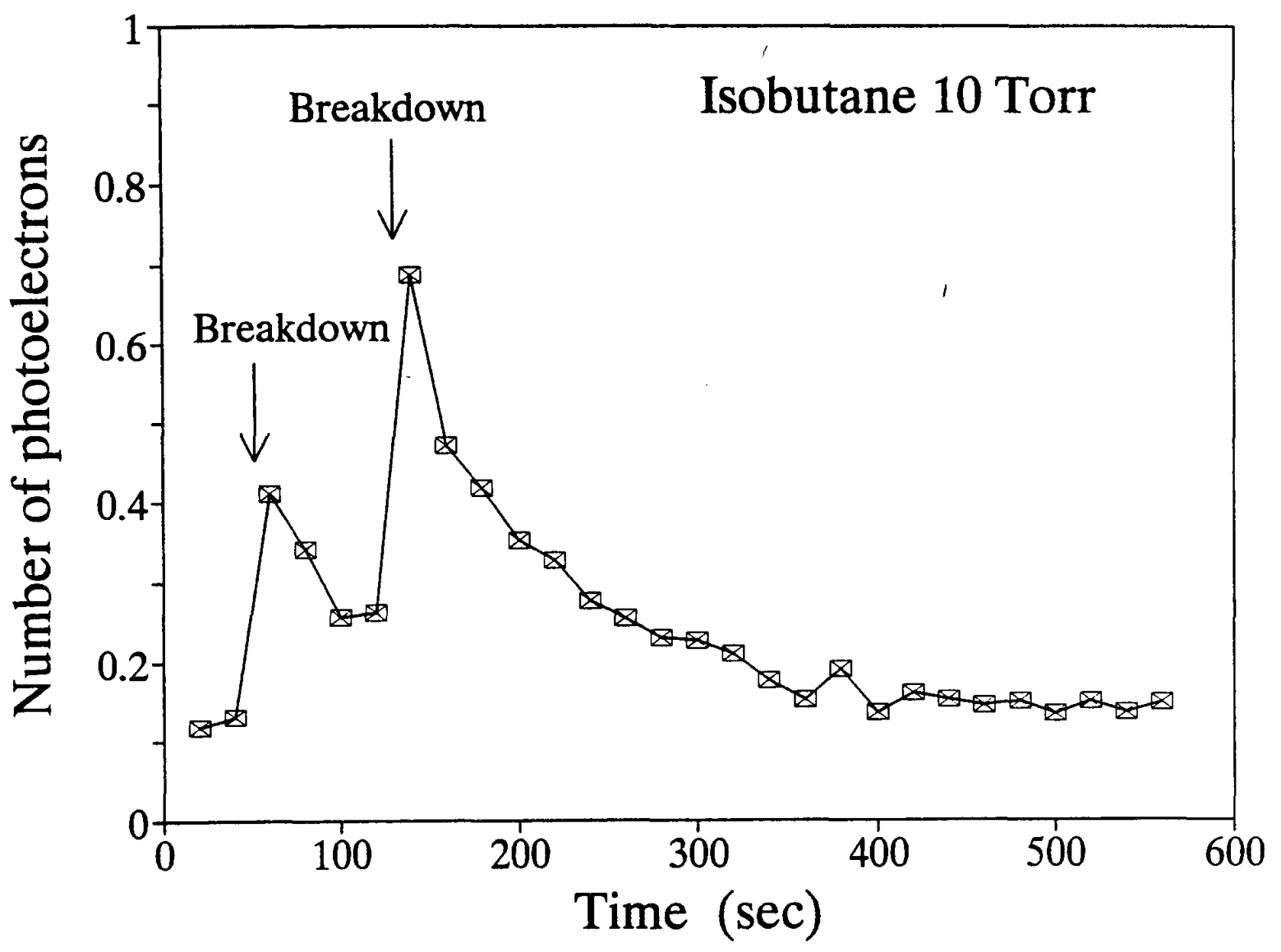

Fig. 3 


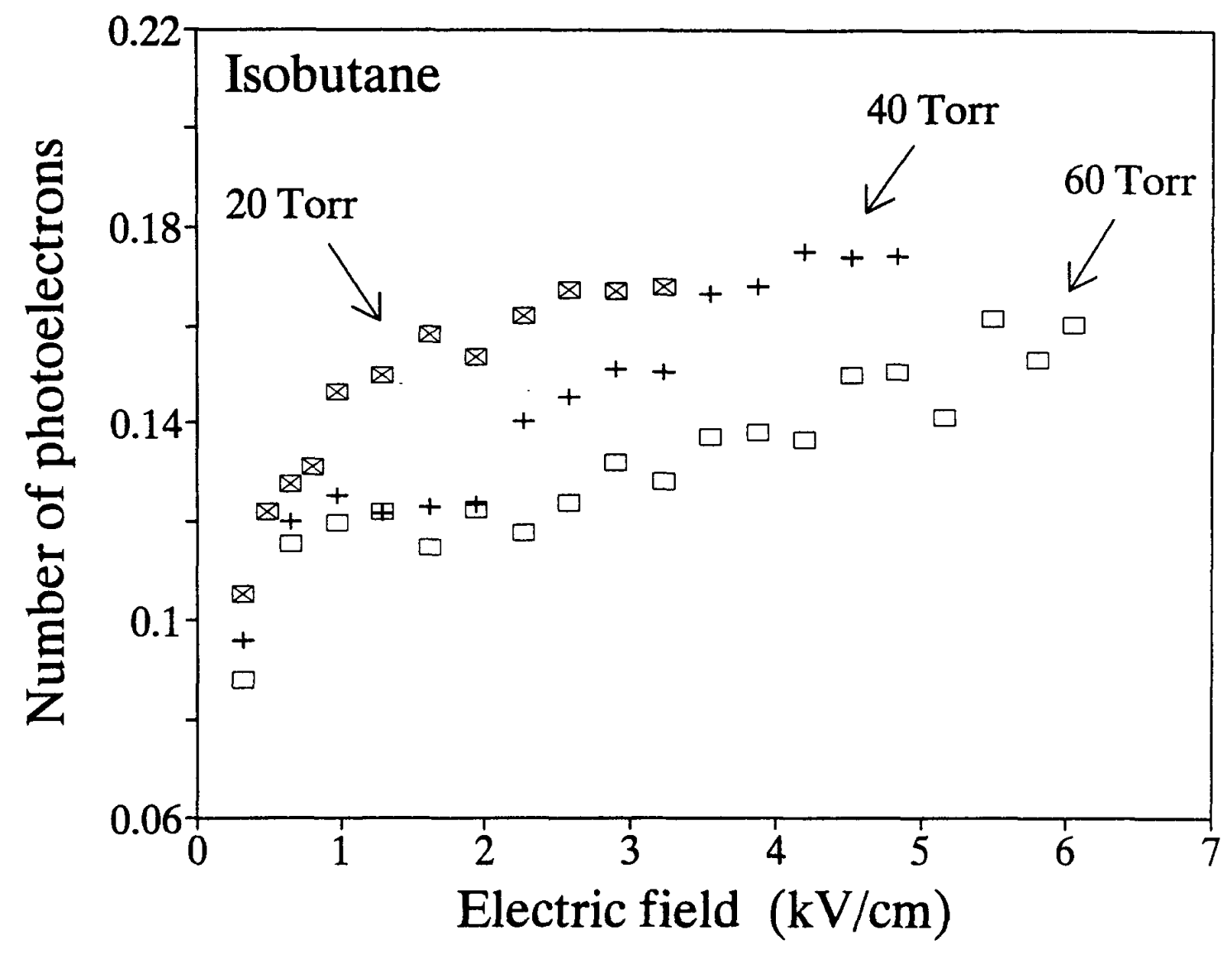

Fig. 4 

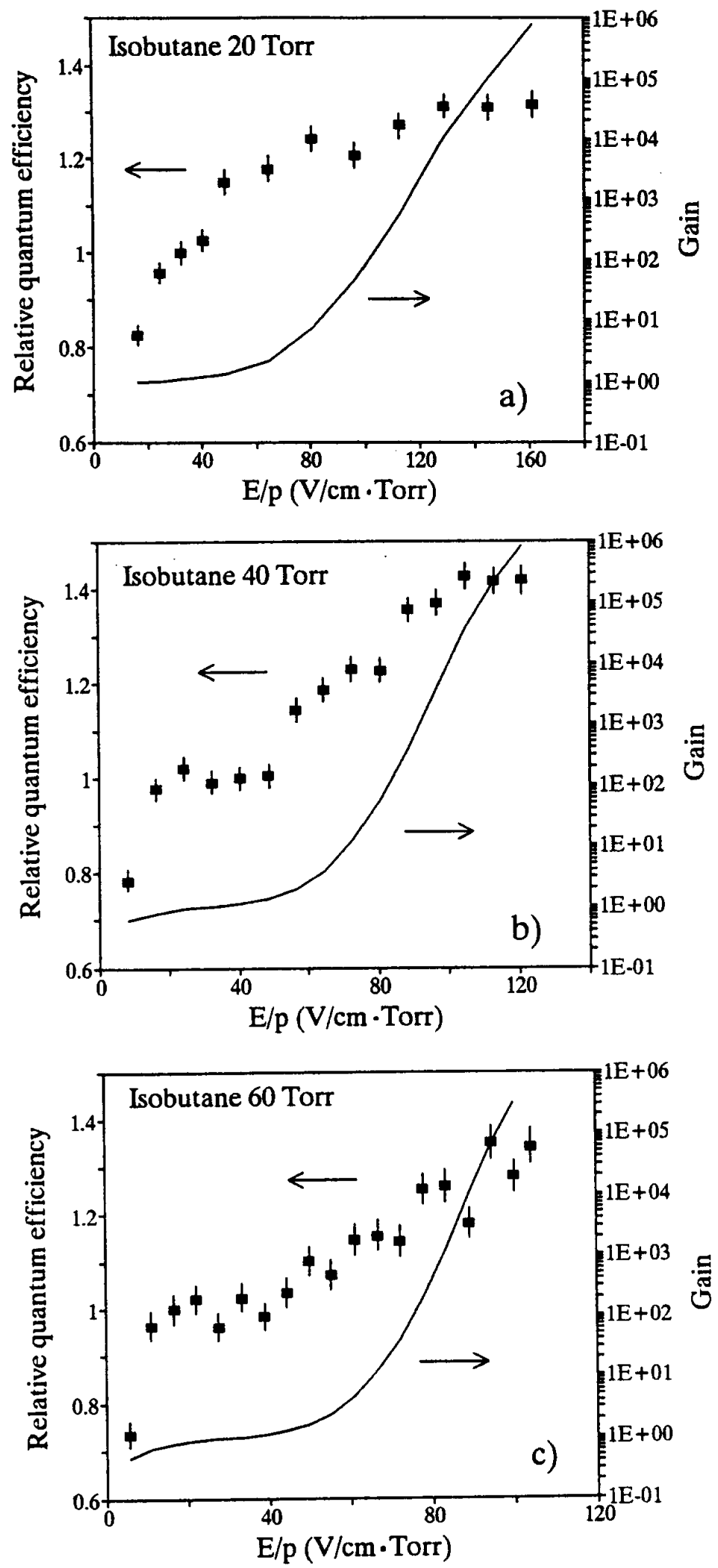

Fig. 5 

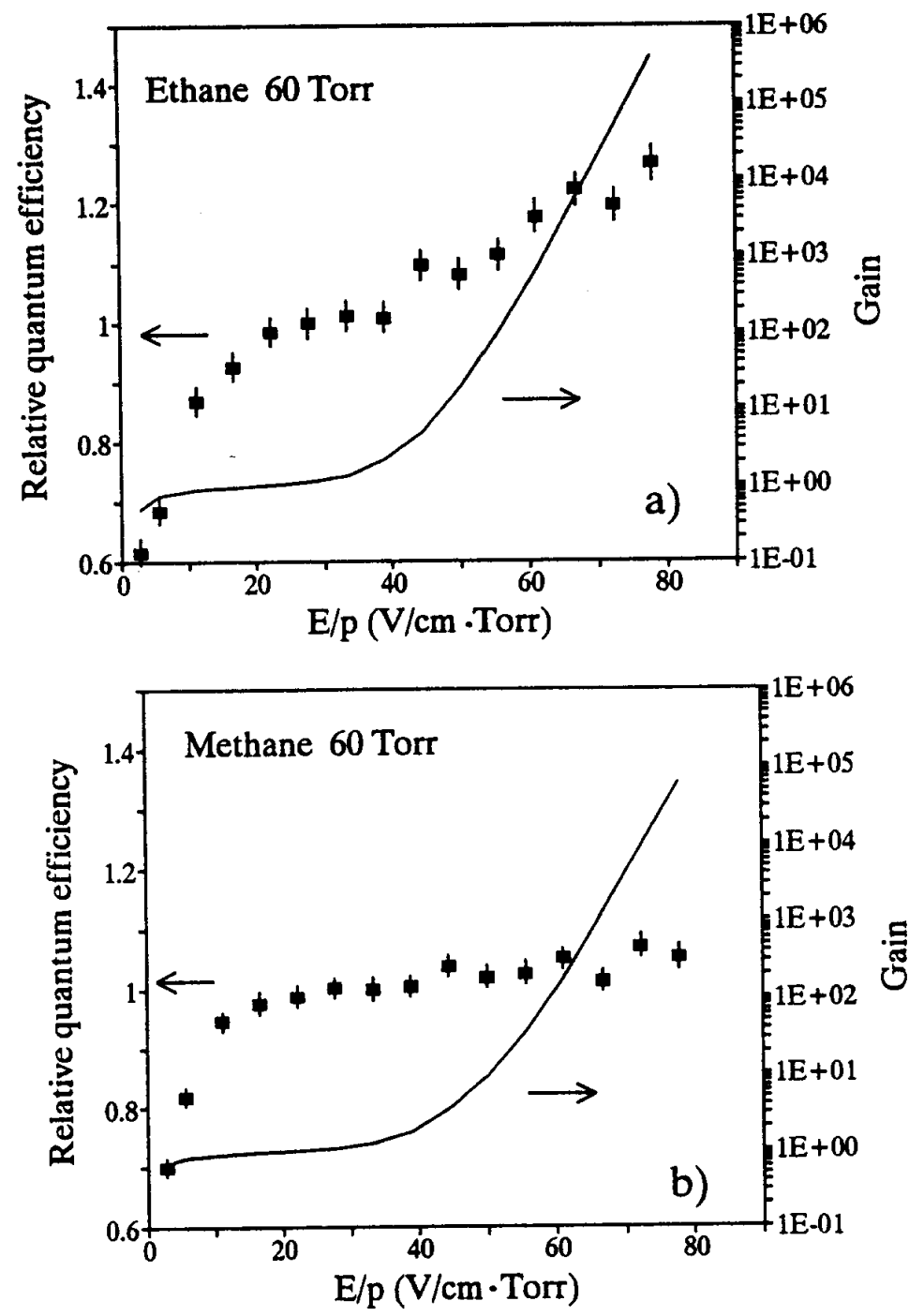

Fig. 6 


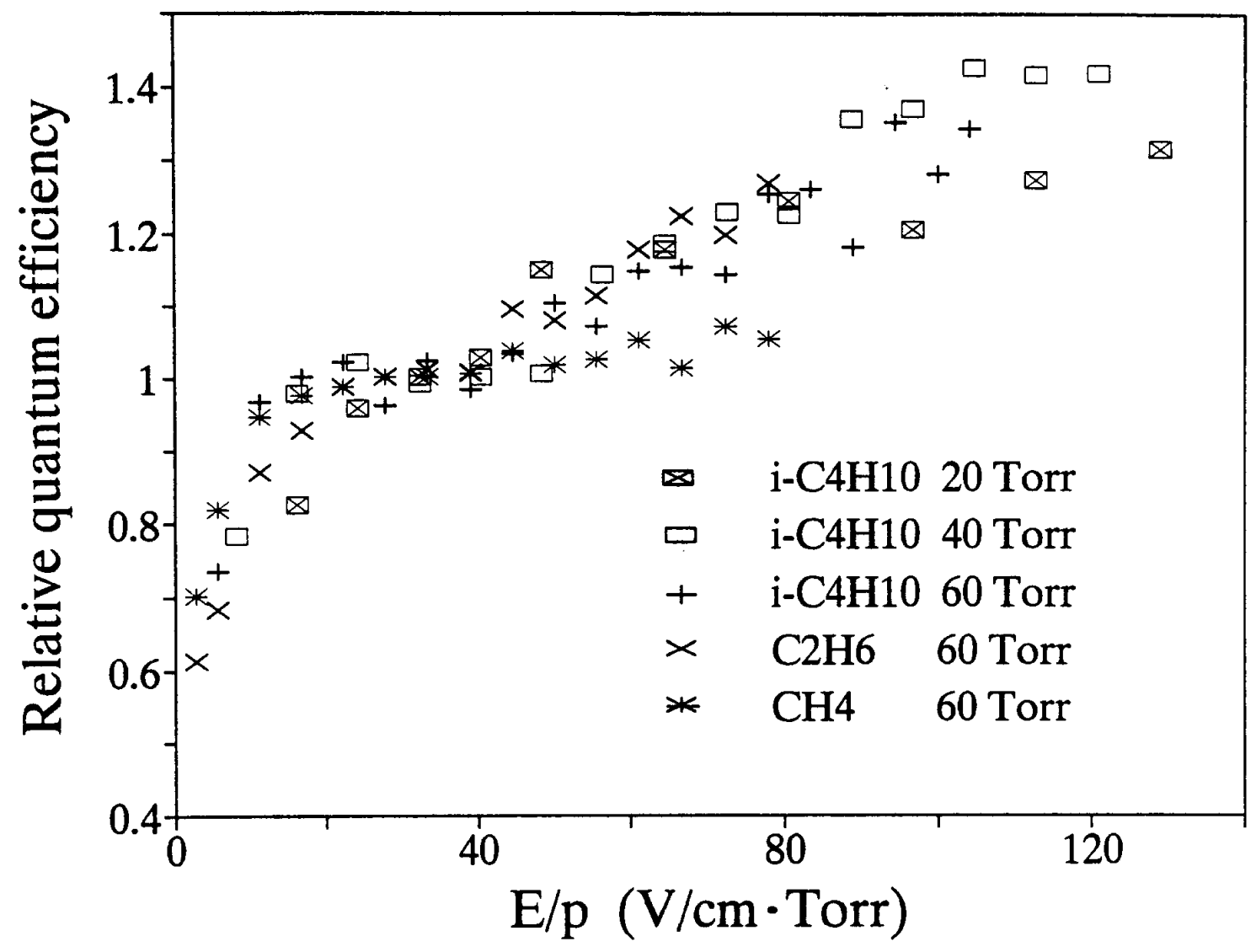

Fig. 7 


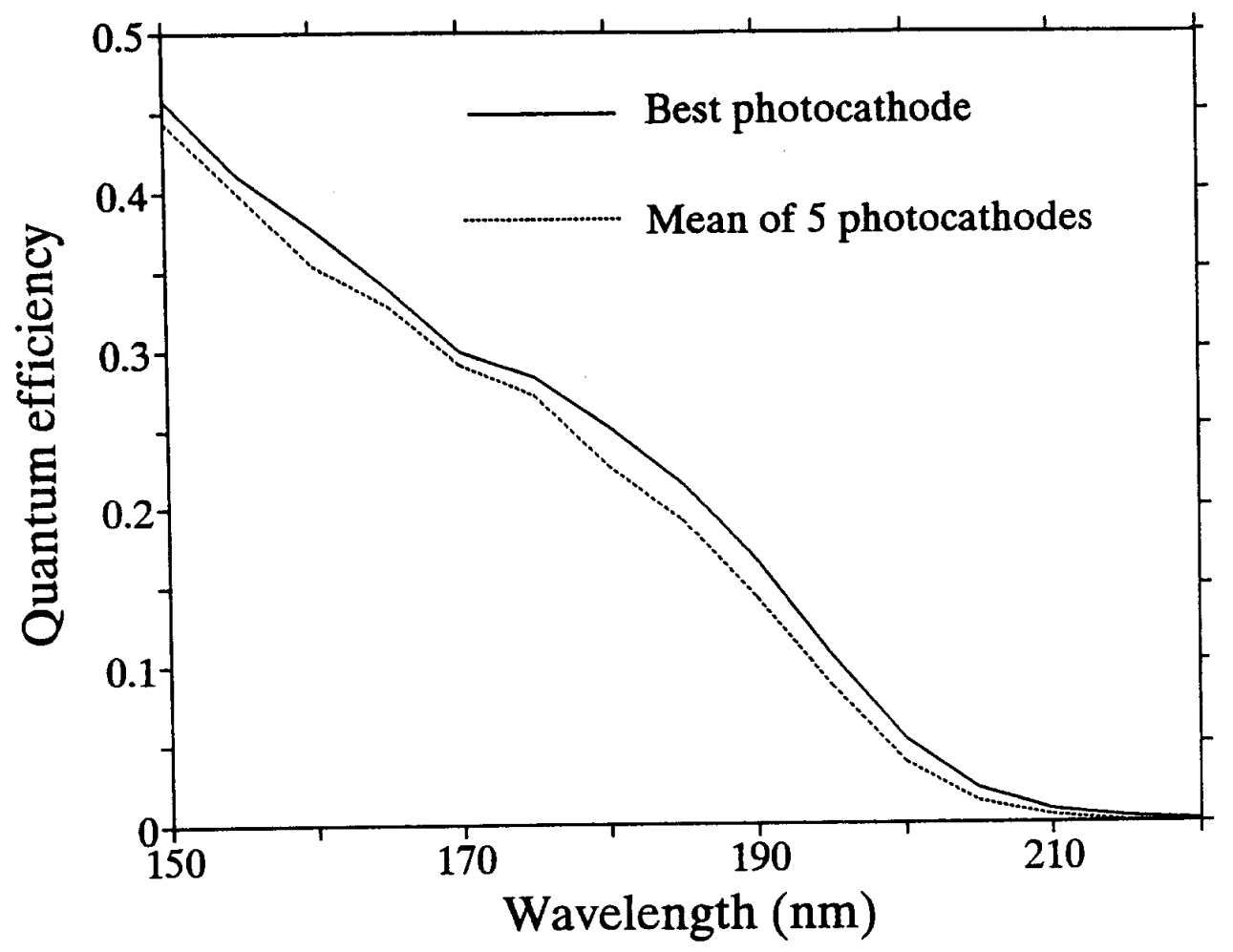

Fig. 8

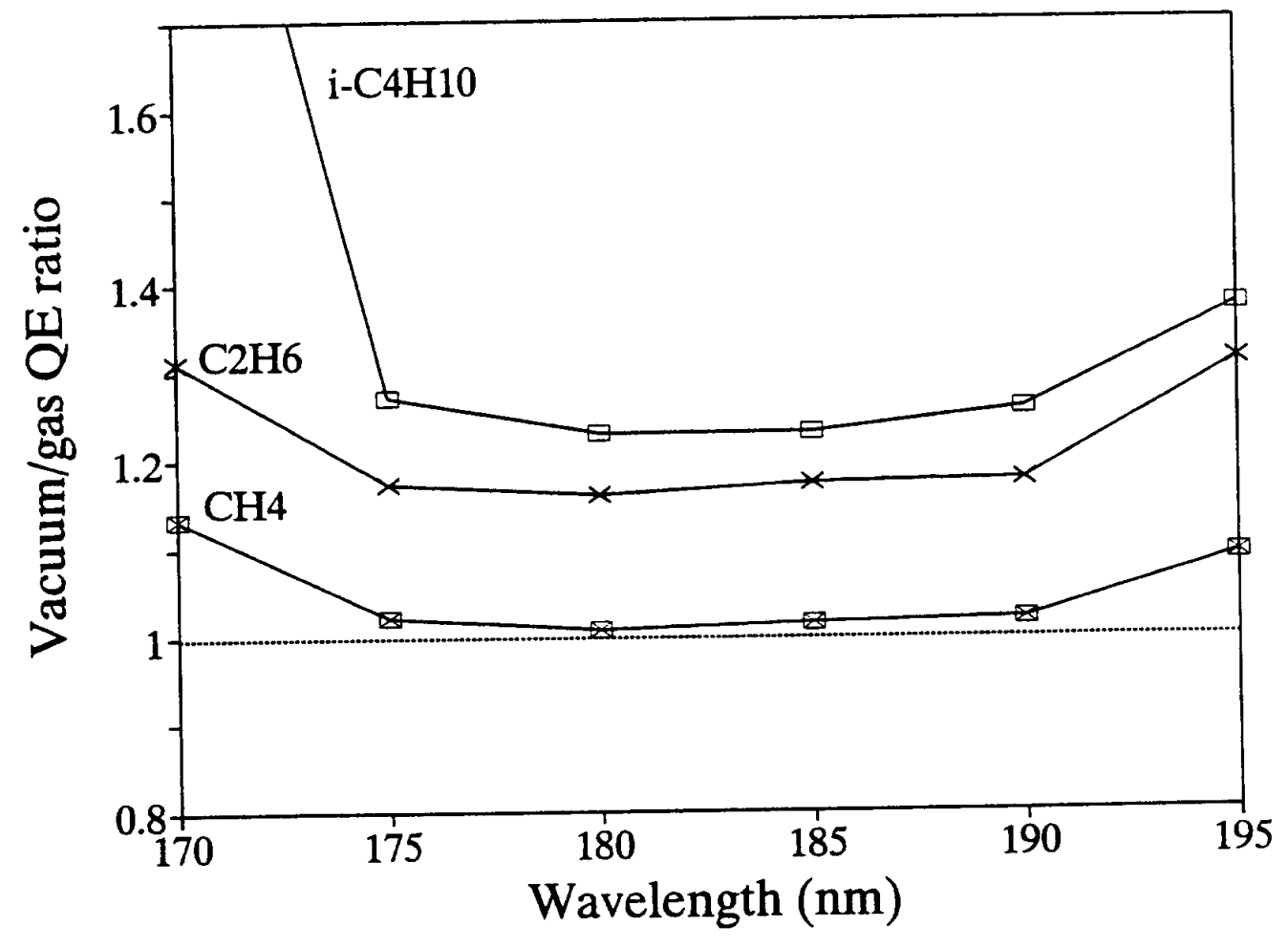

Fig. 9 


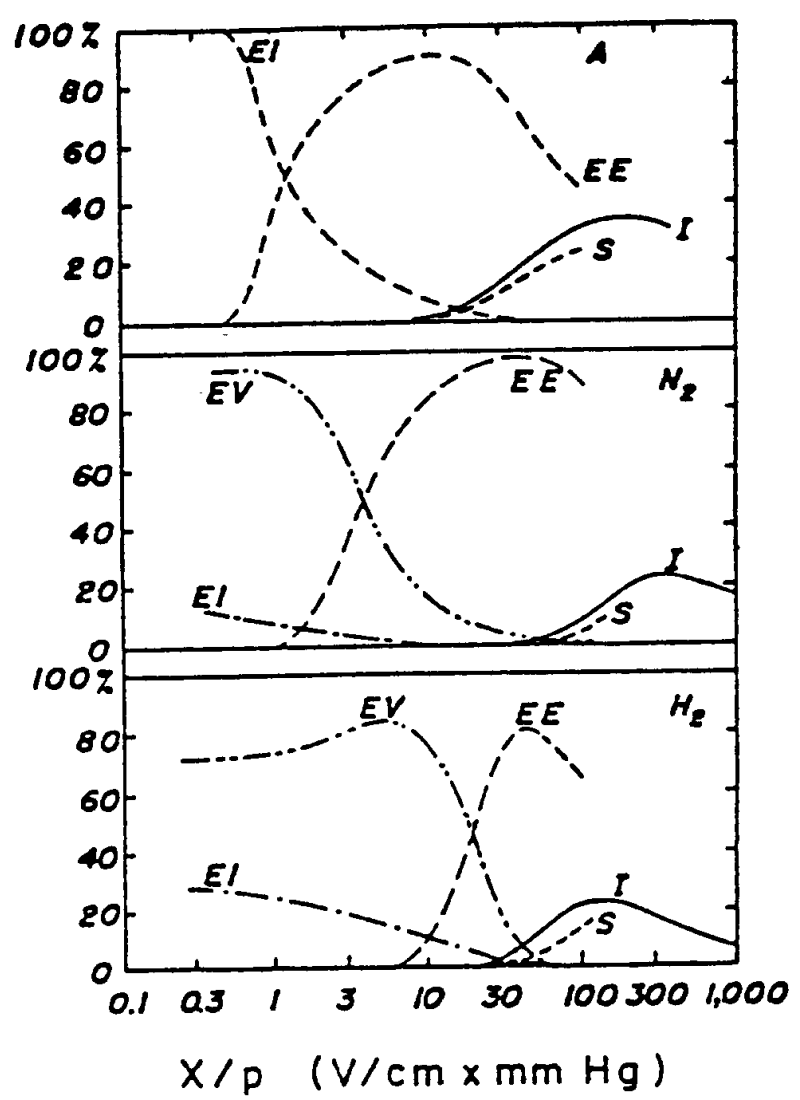

Fig. 10 
